\title{
Explicit Spectral Decimation for a Class of Self-Similar Fractals
}

\author{
Sergio A. Hernández and Federico Menéndez-Conde \\ Centro de Investigación en Matemáticas, Universidad Autónoma del Estado de Hidalgo, 42184 Pachuca, HGO, Mexico \\ Correspondence should be addressed to Federico Menéndez-Conde; fmclara@uaeh.edu.mx
}

Received 6 September 2012; Revised 22 December 2012; Accepted 3 January 2013

Academic Editor: Jiaxin $\mathrm{Hu}$

Copyright (c) 2013 S. A. Hernández and F. Menéndez-Conde. This is an open access article distributed under the Creative Commons Attribution License, which permits unrestricted use, distribution, and reproduction in any medium, provided the original work is properly cited.

The method of spectral decimation is applied to an infinite collection of self-similar fractals. The sets considered are a generalization of the Sierpinski Gasket to higher dimensions; they belong to the class of nested fractals and are thus very symmetric. An explicit construction is given to obtain formulas for the eigenvalues of the Laplace operator acting on these fractals.

\section{Introduction}

In 1989, J. Kigami [1] gave an analytic definition of a Laplace operator acting on the Sierpinski Gasket; a few years later, this definition was extended to include the Laplacians on a large class of self-similar fractal sets [2], known as postcritically finite sets (PCF sets). The method of spectral decimation introduced by Fukushima and Shima in the 1990s provides a way to evaluate the eigenvalues of Kigami's Laplacian. In general terms, this method consists in finding the eigenvalues of the self-similar fractal set by taking limits of eigenvalues of discrete Laplacians that act on some graphs that approximate the fractal. The spectral decimation method was applied in [3] to the Sierpinski Gasket, in order to give an explicit construction which allows one to obtain the set of eigenvalues. In [4], it was shown that it is possible to apply the spectral decimation method to a large collection of p.c.f. sets, including the family of fractals known as nested fractals that was introduced by T. Lindstrøm in [5]. In addition to the Sierpinski Gasket, the spectral decimation method has been applied in several specific cases of p.c.f. fractals (e.g., [610]); also, the method has been proved useful to study the spectrum of particular fractals that are not p.c.f. (e.g., $[11,12]$ ) and of fractafolds modeled on the Sierpinski Gasket [13]. The spectral decimation method has also shown to be a very useful tool for the analysis of the structure of the spectra of the Laplacians of some fractals (e.g., [14-16]).

In the present work, we develop in an explicit way the spectral decimation method for an infinite collection of self-similar sets that we will denote by $\mathbf{P}_{n}(n \geq 2$ a positive integer). The definition of these sets is given in Definition 1. For the cases $n=2,3$, they correspond, respectively, to the unit interval and the Sierpinski Gasket. For larger values of $n$ they give a quite natural extension of the Sierpinski Gasket to higher dimensions. The spectral decimation method for the cases $n=2,3$ is presented with thorough detail in [17]. Our presentation follows this reference to some extent. However, some technical difficulties arise for $n \geq 4$. This is mainly due to the fact that-even though the fractals considered are very symmetric-the graphs approximating the fractal are not as homogeneous as the ones approximating the Sierpinski Gasket. For instance, if we consider the graph obtained by taking away the boundary points from $\Gamma_{1}$ (see Definition 2 and Figures 1 and 2), then it will be a complete graph only for $n \leq 3$. A consequence of this is the appearance of sets of two types of vertices that have to be dealt with separately and which we denote by $F_{r, s}$ and $G_{r, s}$; for $n \leq 3$, the sets $G_{r, s}$ are empty. We also make the observation that the approximating graphs $\Gamma_{k}$ are nonplanar when $n>3$.

In Section 2, we present general facts about self similar sets, for the sake of completeness and in order to establish notation. In Section 3, we introduce the sets $\mathbf{P}_{n}$ that are the subject of study in this work; at the end of the section, we find the Hausdorff dimension of these fractals when embedded in the Euclidean space. In Section 4, we define the graphs that approximate the self-similar sets $\mathbf{P}_{n}$ and fix more notations. Our main result is presented in Section 5 (Theorem 3); it is shown that the eigenvalues and eigenfunctions of the 


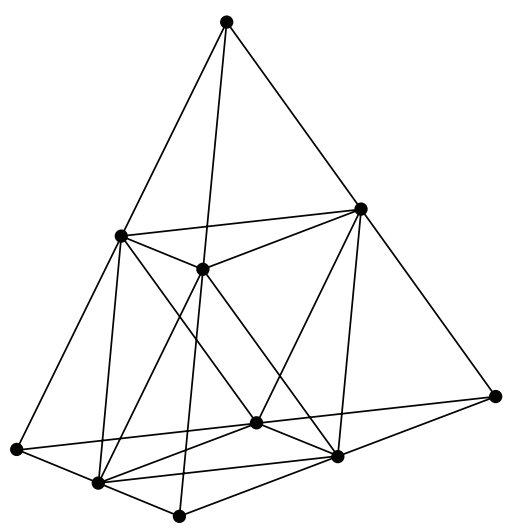

FIgURE 1: The first approximating graph $\Gamma_{1}$ for the fractal $\mathbf{P}_{4}$.

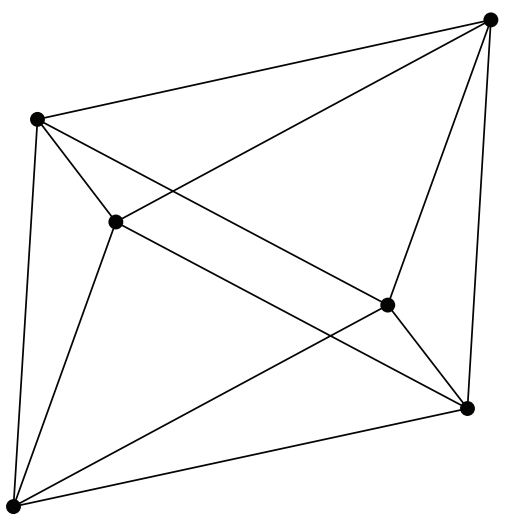

FIgURE 2: The graph $\Gamma_{1}$ for the fractal $\mathbf{P}_{4}$, minus the boundary points.

discrete Laplacians of the approximating graphs can be obtained recursively. Finally, in Section 6, it is shown that the eigenvalues of the Laplace operator in $\mathbf{P}_{n}$ can be recovered by taking limits of the discrete Laplacians; in order to do this, we solve the so-called renormalization problem for this case (see Theorem 5).

\section{Notation and Preliminaries}

We denote by $\mathbf{S}_{n}$ the shift space with $n$ symbols. In this work, we will always consider these $n$ symbols to be the numbers $0,1, \ldots, n-1 . S_{n}$ is a compact space (see, e.g., [18]) when equipped with the metric

$$
\delta\left(a_{0} a_{1} a_{2} a_{3} \ldots ; b_{0} b_{1} b_{2} b_{3} \ldots\right)=r^{k}, \quad 0<r<1,
$$

where

$$
k=\min \left\{j \geq 0 \mid a_{j} \neq b_{j}\right\} .
$$

We will use the dot notation $\dot{a}$, meaning that the symbol $a$ repeats to infinity.

Let $\mathbf{x}=x_{0} x_{1} x_{2} \ldots$ be an element of $\mathbf{S}_{n}$ and $a \in\{0, \ldots, n-$ $1\}$. We denote by $T_{a}$ the shift-operator given by

$$
T_{a}(\mathbf{x})=a x_{0} x_{1} x_{2} \ldots
$$

It is easy to see that

$$
\delta(T(\mathbf{x}) ; T(\mathbf{y}))=r \delta(\mathbf{x} ; \mathbf{y}),
$$

so that $T_{a}$ is a contraction (by factor $r$ ). The space $\mathbf{S}_{n}$ is a self-similar set, equal to $n$ smaller copies of itself, with $\left\{T_{0}, \ldots, T_{n-1}\right\}$ the corresponding contractions. Even more so, it can be proved (Theorem 1.2.3. in [18]) that if $K$ is any selfsimilar set, then it is homeomorphic to a quotient space of the form $\mathbf{S}_{n} / \sim$ for a suitable equivalence relation.

For $K=\mathbf{S}_{n} / \sim$ and $a$ a word of length $m$

$$
\mathbf{a}=\left(a_{0} a_{1} \cdots a_{m-1}\right),
$$

denote by $T_{\mathbf{a}}$ the shift operator given by

$$
T_{\mathbf{a}}(\mathbf{x})=a_{0} \cdots a_{m-1} x_{0} x_{1} x_{2} \cdots
$$

The operator $T_{\mathrm{a}}$ is called an $m$-contraction, and the sets of the form $T_{\mathbf{a}}(K)$ are known as the cells of level $m$ of the self-similar set $K$. We note that, for each choice of $m, K$ is the union of the $n^{m}$ cells of level $m$.

\section{The Self-Similar Fractals $\mathbf{P}_{n}$}

Here we will introduce the self-similar fractals $\mathbf{P}_{n}$ that are the subject of analysis in this work.

Definition 1. For $n \in \mathbb{N}$, define $\mathbf{P}_{n}$ as the quotient space $\mathbf{S}_{n} / \sim$, with the equivalence relation given by

$$
a_{0} a_{1} a_{2} \cdots a_{k} b \dot{c} \sim a_{0} a_{1} a_{2} \cdots a_{k} c \dot{b}
$$

for any choice of symbols $a_{j}, b$, and $c$.

$\mathbf{P}_{1}$ is a trivial space with only one element, $\mathbf{P}_{2}$ is homeomorphic to a compact interval in $\mathbb{R}$, and $\mathbf{P}_{3}$ is homeomorphic to the well-known Sierpinski Gasket. For any value of $n, \mathbf{P}_{n}$ can be embedded in the Euclidean space; more precisely, there exists a (quite natural) homeomorphism between $\mathbf{P}_{n}$ and a compact self-similar set $K_{n} \subset R^{n-1}$. Below, we define the sets $K_{n}$; for these representations of $\mathbf{P}_{n}$, we will be able to find their Hausdorff dimensions.

Take $n$ points $x_{0}, \ldots, x_{n-1} \in \mathbb{R}^{n-1}$ that do not lie in the same $(n-2)$-dimensional hyperplane; for $n=3$, those points will be the "vertices" of the Sierpinski Gasket. For $n=4$, the fractal $K_{4}$ will be some sort of a Sierpinski tetrahedron (see Figure 3 ), while the four points $x_{j}$ will be the vertices of the tetrahedron.

Consider the contractions

$$
f_{i}(x)=\frac{x+x_{i}}{2}, \quad i=1, \ldots, n-1
$$

We note that $f_{i}$ maps each $x \in \mathbb{R}^{n-1}$ to the midpoint of $x$ and $x_{i}$ (hence, leaving $x_{i}$ fixed). Define $K_{n}$ as the unique compact set such that

$$
K_{n}=\bigcup_{i=0}^{n-1} f_{i}\left(K_{n}\right)
$$




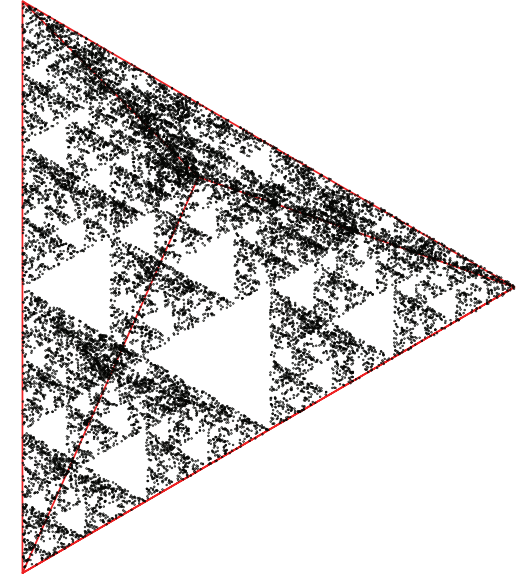

Figure 3: A representation of $K_{4}$, generated with Matlab.

We note that, for $i \neq j$, the sets $f_{i}\left(K_{n}\right)$ and $f_{j}\left(K_{n}\right)$ intersect at exactly one point: $f_{i}\left(x_{j}\right)=f_{j}\left(x_{i}\right)$. From this, it follows that the map $\pi: \mathbf{P}_{n} \rightarrow K_{n}$ given by

$$
\pi\left(\omega_{0} \omega_{1} \omega_{2} \ldots\right)=\bigcap_{m \geq 0} f_{\omega_{0}} \circ f_{\omega_{1}} \circ \cdots \circ f_{\omega_{m}}\left(K_{n}\right)
$$

is a well-defined homeomorphism; also, for every $k=$ $0, \ldots, n-1$, the following diagram commutes (cf. Theorem 1.2.3 in [18]):

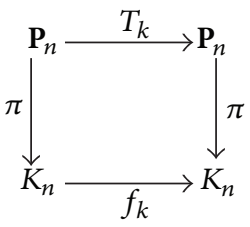

The sets $K_{n}$ satisfy the Moran-Hutchinson open set condition namely, there exists a bounded nonempty open set $O \subset \mathbf{P}_{n}$ such that

$$
\begin{gathered}
f_{i}(O) \subset O, \quad \forall i \in\{0, \ldots, n-1\}, \\
f_{i}(O) \cap f_{j}(O), \quad \forall i \neq j .
\end{gathered}
$$

Just take $O=K_{n} \backslash\left\{x_{0}, \ldots, x_{n-1}\right\}$. From this and the fact that $K_{n}$ is equal to $n$ contractions of itself (by factor $1 / 2$ ), it follows from Moran's theorem (Corollary 1.5.9 in [18]) that the Hausdorff dimension of $K_{n}$, with respect to theEuclidean metric, is equal to $\log n / \log 2$.

We end this section with two relevant notes.

For some values of $n$, it might be possible to embed $K_{n}$ isometrically into the Euclidean space of a dimension $m$ smaller than $n-1$. Of course, the dimension of the fractal gives a restriction to the minimal value of $m$.

The representations $K_{n}$ are somehow useful to visualize the self-similar fractals $\mathbf{P}_{n}$. However, this representation and its metric do not play any role in the analysis carried out in the next sections; we will therefore focus on the more abstract definition of $\mathbf{P}_{n}$ given at the beginning of this section.

\section{Graph Approximations of Self-Similar Sets}

In this and the next sections, we consider the self-similar set $\mathbf{P}_{n}$ defined above, for an arbitrary but fixed value of $n \geq 2$.

Let $V_{0}$ be the set of points in $\mathbf{P}_{n}$ that have the form $\dot{k}$ with $k=1, \ldots, n-1$. We call $V_{0}$ the boundary of $\mathbf{P}_{n}$. Likewise, for $m \in \mathbb{N}$, let $V_{m}$ be the subset of $\mathbf{P}_{n}$ of points of the form $a_{0} \cdots a_{m-1} \dot{k}$. In other words, $x \in V_{m}$ if and only if it belongs to the image of $V_{0}$ under some $m$-contraction.

Next, we define the graphs that will approximate $\mathbf{P}_{n}$.

Definition 2. Denote by $\Gamma_{0}$ the complete graph of $n$ vertices, with $V_{0}$ being its set of vertices. For $m \in \mathbb{N}$, let $\Gamma_{m}$ be the graph with the set of vertices $V_{m}$ and edge relation established by requiring $x$ to be connected with $y$ if and only if there exists an $m$-contraction $T_{\mathbf{a}}$ such that both points $x$ and $y$ are in $T_{\mathbf{a}}\left(V_{0}\right)$.

We can see that an equivalent formulation is that two vertices $x$ and $y$ share an edge in $\Gamma_{m}$ only when their first $m$ symbols coincide. It is worth noting that even though $V_{0} \subset$ $V_{1} \subset V_{2} \ldots$, the edge relation is never preserved; this follows from the fact that if $x \neq y$ are connected in $\Gamma_{m}$, then their $(m+1)$-th symbols cannot be equal, so that they will not be connected in $\Gamma_{m+1}$.

For each $m \in \mathbb{N}$, let $\Delta_{m}$ be the graph Laplacian on $\Gamma_{m}$. We consider the Laplacian as acting on a space with boundary. More precisely, for a real-valued function $u$ defined on $V_{m}$ and $x$ in $V_{m} \backslash V_{0}$,

$$
\Delta_{m} u(x)=\sum_{y \sim x}(u(x)-u(y)),
$$

with the sum over all vertices $y$ that share an edge with $x$; the boundary values remain unchanged. Also, $u$ is an eigenfunction of $\Delta_{0}$ with eigenvalue $\lambda$, if

$$
\Delta_{m} u(x)=\lambda u(x), \quad \forall x \in V_{m} \backslash V_{0} .
$$

We denote by $E_{m}(\cdot, \cdot)$ the associated quadratic form (known as the energy product of the graph):

$$
E_{m}(u, v)=\left(\Delta_{m} u, v\right)=\sum_{x \sim y}(u(x)-u(y))(v(x)-v(y)),
$$

for $u$ and $v$ real-valued functions defined on $V_{m}$ and the sum being taken over the pairs of vertices $(x, y)$ that are connected to each other. Also, we use the abbreviation $E(u)=E(u, u)$.

\section{Spectral Decimation}

Let $m>1$ and suppose $u$ is an eigenfunction of $\Delta_{m-1}$, with eigenvalue $\lambda_{m-1}$. We will show that it is always possible to extend this function to the domain $V_{m}$ so that it will be an eigenfunction of $\Delta_{m}$ (with not the same eigenvalue). 


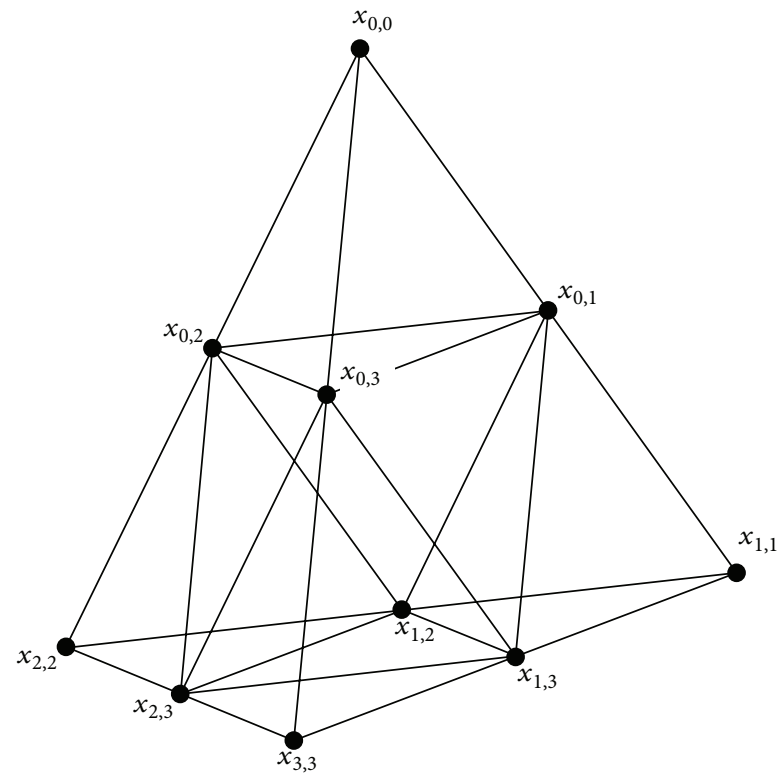

Figure 4: A cell of level $m-1$ of a graph $\Gamma_{m}$, approximating $\mathbf{P}_{4}$.

In order to do this, we will derive necessary conditions for the extension to be an eigenfunction; in the process, it will become clear that those conditions are also sufficient.

Suppose that $u$ is an eigenfunction of $\Delta_{m}$ with eigenvalue $\lambda_{m}$; we aim to write the values of $u_{m}$ in $V_{m} \backslash V_{m-1}$ in terms of its values in $V_{m-1}$. Without loss of generality, we can restrict ourselves to the set $V_{m} \cap T_{\mathbf{a}}\left(\mathbf{P}_{n}\right)$ for a fixed $(m-1)$-contraction $T_{\mathrm{a}}$; this is because the vertices of $\Gamma_{m}$ that belong to the set $\left(V_{m}\right)$ $\left.V_{m-1}\right) \cap T_{\mathbf{a}}\left(\mathbf{P}_{n}\right)$ are not connected to any vertices outside the cell $T_{\mathbf{a}}\left(\mathbf{P}_{n}\right)$. Denote the elements of this set by

$$
x_{b, c}=\mathbf{a} b \dot{c}, \quad b, c=0, \ldots, n-1 .
$$

It is clear that $x_{b, c}=x_{c, b}$ and also that $x_{b, c} \in V_{m-1}$ if and only if $b=c$. This is shown in Figure 4.

For each point $x_{r, s} \in V_{m} \cap T_{\mathbf{a}}\left(\mathbf{P}_{n}\right)$, define the sets of vertices

$$
\begin{aligned}
& F_{r, s}=\left\{x_{i, j} \mid i \neq j,\{r, s\} \cap\{i, j\} \neq \emptyset\right\}, \\
& G_{r, s}=\left\{x_{i, j} \mid i \neq j,\{r, s\} \cap\{i, j\}=\emptyset\right\} .
\end{aligned}
$$

In other words, $F_{r, s}$ is the set of vertices (not in $\Gamma_{m-1}$ ) that are connected to the vertex $x_{r, s}$ in $\Gamma_{m}$, and $G_{r, s}$ is the set of vertices (not in $\Gamma_{m-1}$, either) that are not connected to it.

In the case $n=4$, the graph $\Gamma_{m} \backslash \Gamma_{m-1}$ is an octahedron; hence, for each pair $\{r, s\}$, the subgraph determined by the vertices in $F_{r, s}$ is a 4-cycle one, while $G_{r, s}$ consists of a single vertex (the one opposite to $x_{r, s}$ in the octahedron). For general $\mathbf{P}_{n}$, we can see that the following.

The graph $\Gamma_{m} \backslash \Gamma_{m-1}$ has $n(n-1)$ vertices, all of them with degree $2(n-2)$. Each of these vertices is connected to another 2 vertices in $\Gamma_{m-1}$.

The subgraph determined by $F_{r, s}$ consists of two complete graphs, each one with $n-2$ vertices. The two

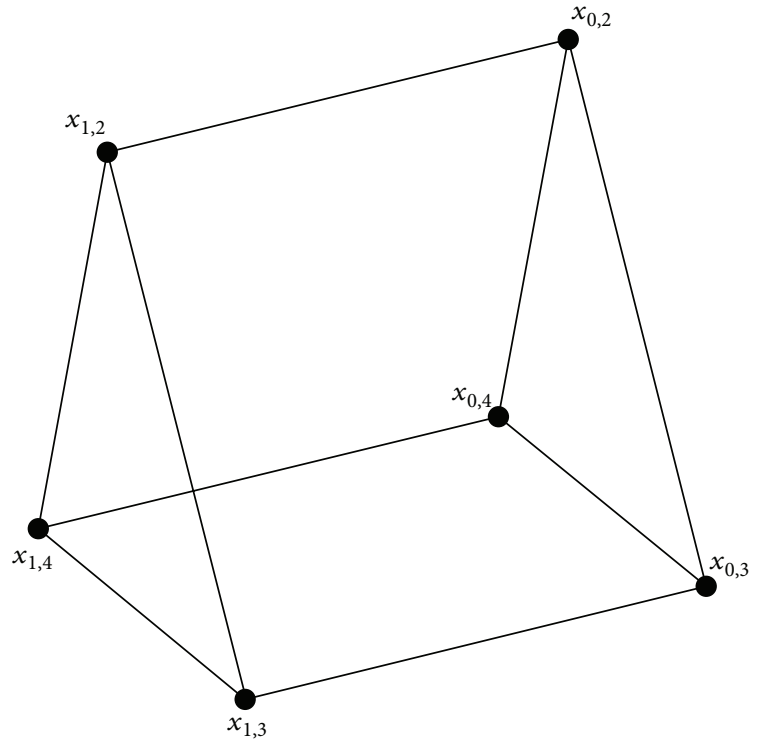

Figure 5: The graph determined by $F_{0,1}$ in a graph $\Gamma_{m}$, approximating $\mathbf{P}_{5}$.

complete graphs are joined to each other pairwise, thus forming a "prism," (a true prism only in the case $n=5$, where the base is a 3-cycle one, as shown in Figure 5).

The subgraph determined by $G_{r, s}$ has $(n-2)(n-3) / 2$ vertices, each one of them with degree $2(n-4)$.

In $\Gamma_{m}$, each vertex that belongs to $G_{r, s}$ is connected to exactly four vertices in $F_{r, s}$. On the other hand, each vertex that belongs to $F_{r, s}$ is connected to $n-2$ vertices in $G_{r, s}$.

Now, having noted all that, we proceed with the calculations. For every $r \neq s$ we have

$$
\begin{aligned}
\left(2(n-1)-\lambda_{m}\right) u\left(x_{r, s}\right)= & u\left(x_{r, r}\right)+u\left(x_{s, s}\right) \\
& +\sum_{F_{r, s}} u\left(x_{i, j}\right) .
\end{aligned}
$$

Adding this up over all the possible values of $r$ and $s$ and rearranging terms yields

$$
\left(2-\lambda_{m}\right) \sum_{r \neq s} u\left(x_{r, s}\right)=(n-1) \sum_{j=0}^{n-1} u\left(x_{j, j}\right)
$$

which for any fixed $a \neq b$ can also be written in the form

$$
\begin{aligned}
& \left(2-\lambda_{m}\right)\left(u\left(x_{a, b}\right)+\sum_{F_{a, b}} u\left(x_{i, j}\right)+\sum_{G_{a, b}} u\left(x_{i, j}\right)\right) \\
& =(n-1) \sum_{j=0}^{n-1} u\left(x_{j, j}\right) .
\end{aligned}
$$


This, together with (18), allows us to express the sum of the values in $G_{a, b}$ in terms of $u\left(x_{a, b}\right)$ and the values at points in $V_{m-1}$; namely, provided $\lambda_{m} \neq 2$, we have that

$$
\begin{aligned}
\sum_{G_{a, b}} u\left(x_{i, j}\right)= & u\left(x_{a, a}\right)+u\left(x_{b, b}\right)-\left(2 n-1-\lambda_{m}\right) u\left(x_{a, b}\right) \\
& +\frac{(n-1)}{2-\lambda_{m}} \sum_{j=0}^{n-1} u\left(x_{j, j}\right) .
\end{aligned}
$$

Next, we will take the sum of the same terms, but only over the $x_{i, j}$ inside the set $F_{a, b}$ for fixed values $a \neq b$. Since $F_{a, b}$ contains two complete graphs with $n-1$ vertices and these complete graphs are pairwise connected to each other, it is clear that each $x_{i, j} \in F_{a, b}$ is connected to other $n-1$ vertices in $F_{a, b}$. Also, recall that each $x_{i, j} \in G_{a, b}$ is connected to exactly four vertices in $F_{a, b}$. For the vertices in $V_{m-1}$, we note that $x_{j, j}$ is connected to $n-2$ vertices in $x_{i, j} \in F_{a, b}$ if $j=a, b$ and to only two vertices otherwise.

From the preceding discussion, it follows the equality

$$
\begin{aligned}
\left(n-\lambda_{m}\right) \sum_{F_{a, b}} u\left(x_{i, j}\right)=4 \sum_{G_{a, b}} u\left(x_{i, j}\right) \\
+(n-2)\left(u\left(x_{a, a}\right)+u\left(x_{b, b}\right)\right) \\
+2(n-2) u\left(x_{a, b}\right)+2 \sum_{j \neq a, b} u\left(x_{j, j}\right) .
\end{aligned}
$$

Consider the expression given by (18) for $\{a, b\}=\{r, s\}$, multiply it by $n-\lambda_{m}$, and substitute equality (22) into it; this gives after arranging terms

$$
\begin{aligned}
& \left(\lambda_{m}^{2}-(3 n-2) \lambda_{m}+2\left(n^{2}-2 n+2\right)\right) u\left(x_{a, b}\right)=4 \sum_{G_{a, b}} u\left(x_{i, j}\right) \\
& \quad+2 \sum_{j \neq a, b} u\left(x_{j, j}\right)+\left(2(n-1)-\lambda_{m}\right)\left(u\left(x_{a, a}\right)+u\left(x_{b, b}\right)\right) .
\end{aligned}
$$

We want to get rid of the terms corresponding to $G_{a, b}$, so we replace it by (21). After straightforward computations, we can see that for $\lambda_{m} \neq 2$

$$
\begin{aligned}
\left(\lambda_{m}^{2}-\right. & \left.(3 n+2) \lambda_{m}+2 n(n+2)\right) u\left(x_{a, b}\right) \\
= & \left(\lambda_{m}^{2}-2(n+2)+8 n\right)\left(u\left(x_{a, a}\right)+u\left(x_{b, b}\right)\right) \\
& +2\left(2 n-\lambda_{m}\right) \sum_{j \neq a, b} u\left(x_{j, j}\right)\left(2-\lambda_{m}\right)^{-1} .
\end{aligned}
$$

The quadratic equation for $\lambda_{m}$ in the left-hand side has roots $n+2$ and $2 n$. The one in the right-hand side has roots 4 and $2 n$. This gives us the following expression for $u\left(x_{a, b}\right)$ in terms of the values of $u$ in $V_{m-1}$ :

$$
u\left(x_{r, s}\right)=\frac{\left(4-\lambda_{m}\right)\left(u\left(x_{r, r}\right)+u\left(x_{s, s}\right)\right)+2 \sum_{j \neq r, s} u\left(x_{j, j}\right)}{\left(2-\lambda_{m}\right)\left((n+2)-\lambda_{m}\right)},
$$

valid for any eigenvalue $\lambda_{m} \neq 2, n+2,2 n$.
For $\lambda_{m}=0$, this reduces to

$$
u\left(x_{r, s}\right)=\frac{2}{n+2}\left(u\left(x_{r, r}\right)+u\left(x_{s, s}\right)\right)+\frac{1}{n+2} \sum_{j \neq r, s} u\left(x_{j, j}\right) .
$$

It is clear from the construction that if $u\left(x_{a, b}\right)$ is defined by (25) and $\lambda_{m}$ is given by (35), then we have that

$$
\Delta_{m} u\left(x_{a, b}\right)=\lambda_{m} u\left(x_{a, b}\right) \quad(a \neq b) .
$$

It remains to verify that this is valid as well in $V_{m-1}$. Of course, this cannot be true for arbitrary values of $\lambda_{m}$ but only at most for specific values depending on $\lambda_{m-1}$; we will find those values in what follows.

Take a point in $V_{m-1}$, say

$$
x_{p, p}=\mathbf{a} \dot{p}, \quad \mathbf{a}=a_{0} \cdots a_{m-1} .
$$

Suppose that $a_{k}=q$ is the last symbol in a that is different from $p$; we can assume that such symbol exists, since otherwise $x_{p, p}$ would be in the boundary $V_{0}$. With this, the point $x_{p, p}$ can also be written in the form

$$
x_{p, p}=\mathbf{a}^{\prime} \dot{q}, \quad \mathbf{a}^{\prime}=a_{0} \cdots a_{k-1} p q \cdots q,
$$

with the necessary number of $q$ 's to make $\mathbf{a}^{\prime}$ a word of length $m-1$. Hence, $x_{p, p}$ is in exactly two different $(m-1)$-cells: $T_{\mathbf{a}}\left(\mathbf{P}_{n}\right)$ and $T_{\mathbf{a}^{\prime}}\left(\mathbf{P}_{n}\right)$, corresponding to each one of its two representations.

Denote by $x_{r, s}^{\prime}$ the points in $T_{\mathbf{a}^{\prime}}\left(\mathbf{P}_{n}\right) \cap V_{m}$, defined as in (16) for the points in $T_{\mathbf{a}}\left(\mathbf{P}_{n}\right) \cap V_{m}$; in particular, $x_{p, p}=x_{q, q}^{\prime}$ (see Figure 6). The value of $u$ in the points $x_{r, s}^{\prime}$ is given by the analogue of (25). The vertex $x_{p, p}$ is connected in $\Gamma_{m}$ to the $2(n-1)$ points of the form $x_{p, j}$ and $x_{q, j}^{\prime}$, from which it follows that $u$ is an eigenfunction of $\Delta_{m}$ with eigenvalue $\lambda_{m}$, if and only if (25) holds for all $x_{r, s} \in V_{m} \backslash V_{m-1}$ and the following equality holds for all $x_{p, p} \in V_{m-1}$ :

$$
\left(2(n-1)-\lambda_{m}\right) u\left(x_{p, p}\right)=\sum_{i \neq p, j \neq q}\left(u\left(x_{p, j}\right)+u\left(x_{q, j}^{\prime}\right)\right) .
$$

On the other hand, since we know that $u$ is an eigenfunction of $\Delta_{m-1}$ with eigenvalue $\lambda_{m-1}$, we also have that

$$
\left(2(n-1)-\lambda_{m-1}\right) u\left(x_{p, p}\right)=\sum_{i \neq p, j \neq q}\left(u\left(x_{i, i}\right)+u\left(x_{j, j}^{\prime}\right)\right) .
$$

Replacing each term in the right-hand side of (30) by its expression given by (25), we can see that

$$
\begin{aligned}
& \left(2(n-1)-\lambda_{m}\right) u\left(x_{p, p}\right) \\
& =2(n-1)\left(4-\lambda_{m}\right) u\left(x_{p, p}\right)+\left(2 n-\lambda_{m}\right)\left(2-\lambda_{m}\right)^{-1} \\
& \quad \times\left((n+2)-\lambda_{m}\right)^{-1},
\end{aligned}
$$




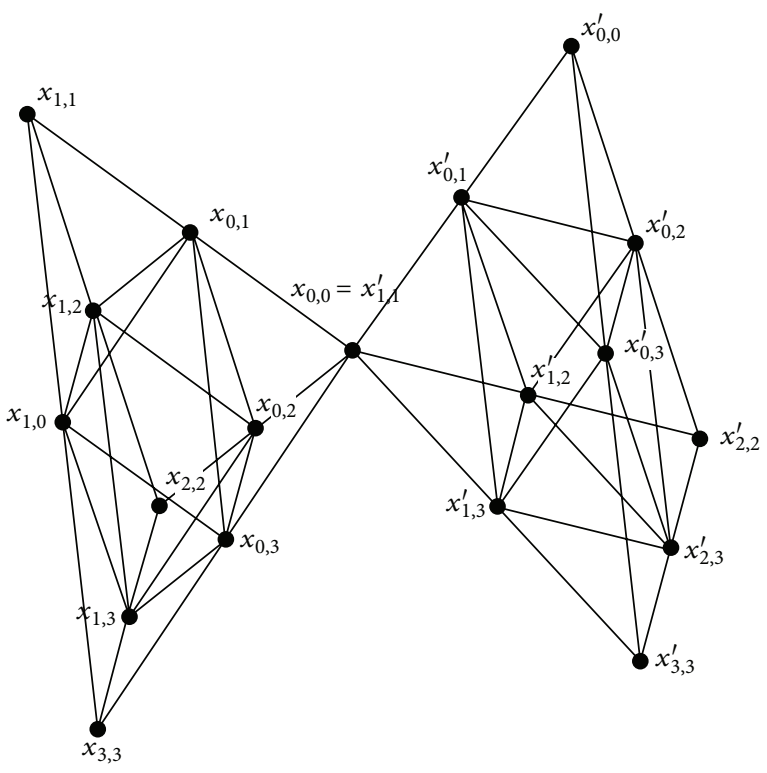

Figure 6: Two cells of level $m-1$ intersecting in a vertex of $\Gamma_{m}$.

and, using (31), this gives

$$
\begin{aligned}
& u\left(x_{p, p}\right) \\
& =\left[2(n-1)\left(4-\lambda_{m}\right)+\left(2 n-\lambda_{m}\right)\left(2(n-1)-\lambda_{m}\right)\right] \\
& \quad \times u\left(x_{p, p}\right)\left(2(n-1)-\lambda_{m}\right)^{-1}\left(2-\lambda_{m}\right)^{-1} \\
& \quad \times\left((n+2)-\lambda_{m}\right)^{-1} .
\end{aligned}
$$

Taking $u\left(x_{p, p}\right) \neq 0$ and cancelling out, after computations, the above equality reduces to the quadratic

$$
\lambda_{m}^{2}-(n+2) \lambda_{m}+\lambda_{m-1}=0
$$

which in turn gives the following recursive characterization of the eigenvalues:

$$
\lambda_{m}=\frac{(n+2) \pm \sqrt{(n+2)^{2}-4 \lambda_{m-1}}}{2} .
$$

Since this procedure can be reversed, we have proved the following result.

Theorem 3. Let $\lambda_{m} \neq 2, n+2$, and $2 n$, and let $\lambda_{m-1}$ be given by (34). Suppose $u$ is an eigenfunction of $\Delta_{m-1}$ with eigenvalue $\lambda_{m-1}$. Extend $u$ to $V_{m}$ by (25). Then $u$ is an eigenfunction of $\Delta_{m}$ with eigenvalue $\lambda_{m}$. Conversely, if $u$ is an eigenfunction of $\Delta_{m}$ with eigenvalue $\lambda_{m} \neq 2, n+2$, and $2 n$, then its restriction to $V_{m-1}$ is an eigenfunction of $\Delta_{m-1}$ with eigenvalue $\lambda_{m-1}$.

\section{The Laplacian on the Self-Similar Fractals}

In order to define the Laplace operator of a p.c.f. fractal by means of graph approximations, it is required to solve the so-called renormalization problem for the fractal (e.g.,
[17], Chapter 4); roughly, this consists in normalizing the graph energies in $\Gamma_{m}$ in order to obtain a self-similar energy in the fractal by taking the limit. This can be achieved if the energies are such that they remain constant for each harmonic extension from $\Gamma_{m}$ to $\Gamma_{m+1}$. Below, we do this for the $\mathbf{P}_{n}$ sets.

Definition 4. For a given function $u$ with domain $V_{m-1}$, we call the extension of $u$ to $V_{m}$ given by (26) its harmonic extension.

The next result gives the explicit solution of the renormalization problem for $\mathbf{P}_{n}$.

Theorem 5. Let $u: V_{m-1} \rightarrow \mathbb{R}$ be arbitrary, and let $u^{\prime}:$ $V_{m} \rightarrow \mathbb{R}$ be its harmonic extension. Then

$$
E_{m}\left(u^{\prime}\right)=\frac{n}{n+2} E_{m-1}(u)
$$

Proof. Note that the energy at level $k$ of a given function equals the sum of the energies at all the $k^{\prime}$-cells for any $k^{\prime} \leq k$, since different cells share no edges. This allows us to restrict ourselves to one fixed $m-1$-cell both while considering $E_{m}\left(u^{\prime}\right)$ and $E_{m-1}(u)$. We use the notation of Section 4 for the vertices of $\Gamma_{m}$ in this cell and write $\widetilde{E}$ for the energy restricted to this cell. We can readily see that

$$
\begin{aligned}
\widetilde{E}_{m-1}(u) & =\sum_{i \neq j}\left(u\left(x_{i, i}\right)-u\left(x_{j, j}\right)\right)^{2} . \\
& =(n-1) \sum_{i=0}^{n-1} u^{2}\left(x_{i, i}\right)-2 \sum_{i \neq j} u\left(x_{i, i} u\left(x_{i}, j\right)\right) .
\end{aligned}
$$

In order to evaluate the energy $\widetilde{E}_{m}$, we consider first the edges joining vertices in $V_{m-1}$ with vertices in $V_{m} \backslash V_{m-1}$ : the edge joining the vertex $x_{a, a}$ with the vertex $x_{a, k}$ contributes to the energy by

$$
\begin{aligned}
& \left(u\left(x_{a, a}\right)-u\left(x_{a, k}\right)\right)^{2} \\
& \quad=\frac{1}{(n+2)^{2}}\left(n u\left(x_{a, a}\right)-2 u\left(x_{k, k}\right)-\sum_{j \neq a, k} u\left(x_{j, j}\right)\right)^{2} .
\end{aligned}
$$

When adding up this over all possible pairs $a \neq k$, each $x_{r, r}$ will appear $n-1$ times as the $x_{a, a}$, another $n-1$ times as the $x_{k, k}$, and $(n-1)(n-2)$ as one of the $x_{j, j}$ 's. Each pair double product $2 x_{r, r} x_{s, s}$ will appear twice for $\{r, s\}=\{a, k\}, 2(n-2)$ times for $\{r, s\}=\{a, j\}$ for some $j$, also $2(n-2)$ times for $\{r, s\}=\{k, j\}$ for some $j$, and finally $(n-2)(n-3)$ times when both $r$ and $s$ are one of the $j$ 's. All this implies 
that the contribution to the energy from these edges is, after simplification,

$$
\begin{aligned}
\sum_{a \neq k}\left(u\left(x_{a, a}\right)-u\left(x_{a, k}\right)\right)^{2} & \\
= & \frac{(n-1)\left(n^{2}+n+2\right)}{(n+2)^{2}} \sum_{i=0}^{n-1} u^{2}\left(x_{i, i}\right) \\
& -\frac{2\left(n^{2}+n+2\right)}{(n+2)^{2}} \sum_{i \neq j} x_{i, i} x_{j, j} .
\end{aligned}
$$

On the other hand, the contribution from the edge that joins the vertices $x_{a, b}$ and $x_{a, c}$ (in $V_{m} \backslash V_{m-1}$ ) equals

$$
\left(u\left(x_{a, b}\right)-u\left(x_{a, c}\right)\right)^{2}=\frac{1}{(n+2)^{2}}\left(u\left(x_{b, b}\right)-u\left(x_{c, c}\right)\right)^{2} .
$$

Taking the sum over all of the vertices in $V_{m} \backslash V_{m-1}$ yields

$$
\begin{aligned}
& \sum_{a \neq b \neq c}\left(u\left(x_{a, b}\right)-u\left(x_{a, c}\right)\right)^{2} \\
& \quad=\frac{(n-1)(n-2)}{(n+2)^{2}} \sum_{i=0}^{n-1} u^{2}\left(x_{i, i}\right)-\frac{2(n-2)}{(n+2)^{2}} \sum_{i \neq j} u\left(x_{i, i}\right) u\left(x_{j, j}\right) .
\end{aligned}
$$

From (39) and (41), it follows that the total energy of the cell is

$$
\widetilde{E}_{m}\left(u^{\prime}\right)=\frac{n(n-1)}{n+2} \sum_{i=0}^{n-1} u^{2}\left(x_{i, i}\right)-2 n(n+2) .
$$

This, together with (37), gives

$$
\widetilde{E}_{m}\left(u^{\prime}\right)=\frac{n}{n+2} \widetilde{E}_{m-1}(u) \text {. }
$$

Taking this result for all the $m-1$-cells concludes the proof.

Definition 6. The energy in $\mathbf{P}_{n}$ is given by

$$
E(u)=\lim _{m \rightarrow \infty}\left(\frac{n+2}{n}\right)^{m} E_{m}(u) \text {. }
$$

The domain of $E(\cdot)$ being the space $D^{(n)}$ of functions such that the energy is finite. Write $D_{0}^{(n)}$ for the subspace of $D^{(n)}$ of functions that vanish on the boundary. The energy product $E(u, v)$ can be recovered by the polarization identity.

Let $\mu$ be a self-similar measure in $\mathbf{P}_{n}$; the Laplacian $\Delta_{\mu}$ is given by the following:

Definition 7 (Kigami's Laplacian). With $\mu$ and $\Delta_{\mu}$ as above, one says that $u$ is in the domain of $\Delta_{\mu}$ if there exists a continuous function $f$ such that

$$
E(u, v)=-\int_{P_{n}} f v d \mu, \quad \forall v \in D_{0}^{(n)}
$$

In such case, we define $\Delta_{\mu} u=f$.
Aside from the above weak representation, a pointwise formula can be obtained for $\Delta_{m} u$, proceeding exactly in the same way as in [17] (Theorem 2.2.1). In the case where $\mu$ is the standard measure in $P_{n}$ (i.e., the only Borel regular measure such that the measure of every $m$-cell is equal to $n^{-m}$ ), the pointwise formula is

$$
\Delta_{\mu} u(x)=\frac{n}{2} \lim _{m \rightarrow \infty}(n+2)^{m} \Delta_{m} u(x) .
$$

This leads to the following: if a sequence $\left\{\lambda_{m}\right\}$ is defined recursively by (35) (assuming that $\lambda_{m}$ is never equal to $n, n+$ 2 , or $2 n$ ) and $u_{m}$ is given by relation (25), then

$$
\lambda=\frac{n}{2} \lim _{m \rightarrow \infty}(n+2)^{m} \lambda_{m}
$$

is an eigenvalue of $\Delta_{\mu}$ with eigenfunction $u$ given by the limit $u_{m} \rightarrow u$. The limit above exists provided that the sign in relation (35) is chosen to be "+" for at most a finite number of times.

\section{Acknowledgments}

The authors are very grateful to Alejandro Butanda and Yolanda Ortega for their valuable assistance with the figures and to the referee for the many useful comments.

\section{References}

[1] J. Kigami, "A harmonic calculus on the Sierpiński spaces," Japan Journal of Applied Mathematics, vol. 6, no. 2, pp. 259-290, 1989.

[2] J. Kigami, "Harmonic calculus on p.c.f. self-similar sets," Transactions of the American Mathematical Society, vol. 335, no. 2, pp. 721-755, 1993.

[3] M. Fukushima and T. Shima, "On a spectral analysis for the Sierpiński gasket," Potential Analysis, vol. 1, no. 1, pp. 1-35, 1992.

[4] T. Shima, "On eigenvalue problems for Laplacians on p.c.f. selfsimilar sets," Japan Journal of Industrial and Applied Mathematics, vol. 13, no. 1, pp. 1-23, 1996.

[5] T. Lindstrøm, "Brownian motion on nested fractals," Memoirs of the American Mathematical Society, no. 420, 1990.

[6] S. Constantin, R. S. Strichartz, and M. Wheeler, "Analysis of the Laplacian and spectral operators on the Vicsek set," Communications on Pure and Applied Analysis, vol. 10, no. 1, pp. 1-44, 2011.

[7] S. Drenning and R. S. Strichartz, "Spectral decimation on Hambly's homogeneous hierarchical gaskets," Illinois Journal of Mathematics, vol. 53, no. 3, pp. 915-937, 2010.

[8] D. J. Ford and B. Steinhurst, "Vibration spectra of the $m$-tree fractal," Fractals, vol. 18, no. 2, pp. 157-169, 2010.

[9] V. Metz, "Laplacians' on finitely ramified, graph directed fractals," Mathematische Annalen, vol. 330, no. 4, pp. 809-828, 2004.

[10] D. Zhou, "Spectral analysis of Laplacians on the Vicsek set," Pacific Journal of Mathematics, vol. 241, no. 2, pp. 369-398, 2009.

[11] N. Bajorin, T. Chen, A. Dagan et al., "Vibration modes of $3 n-$ gaskets and other fractals," Journal of Physics A, vol. 41, no. 1, Article ID 015101, 21 pages, 2008.

[12] N. Bajorin, T. Chen, A. Dagan et al., "Vibration spectra of finitely ramified, symmetric fractals," Fractals, vol. 16, no. 3, pp. 243-258, 2008.

[13] R. S. Strichartz, "Fractafolds based on the Sierpiński gasket and their spectra," Transactions of the American Mathematical Society, vol. 355, no. 10, pp. 4019-4043, 2003. 
[14] K. E. Hare and D. Zhou, "Gaps in the ratios of the spectra of Laplacians on fractals," Fractals, vol. 17, no. 4, pp. 523-535, 2009.

[15] R. S. Strichartz, "Laplacians on fractals with spectral gaps have nicer Fourier series," Mathematical Research Letters, vol. 12, no. 2-3, pp. 269-274, 2005.

[16] D. Zhou, "Criteria for spectral gaps of Laplacians on fractals," The Journal of Fourier Analysis and Applications, vol. 16, no. 1, pp. 76-96, 2010.

[17] R. S. Strichartz, Differential Equations on Fractals: A Tutorial, Princeton University Press, Princeton, NJ, USA, 2006.

[18] J. Kigami, Analysis on Fractals, vol. 143, Cambridge University Press, Cambridge, UK, 2001. 


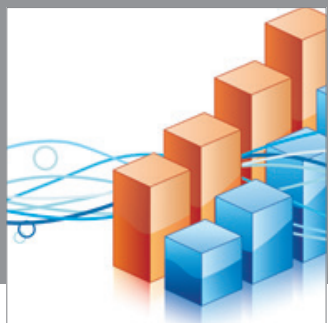

Advances in

Operations Research

mansans

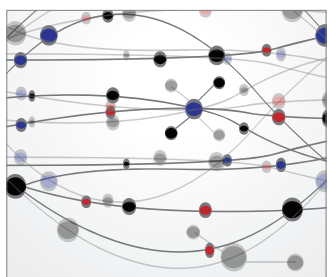

The Scientific World Journal
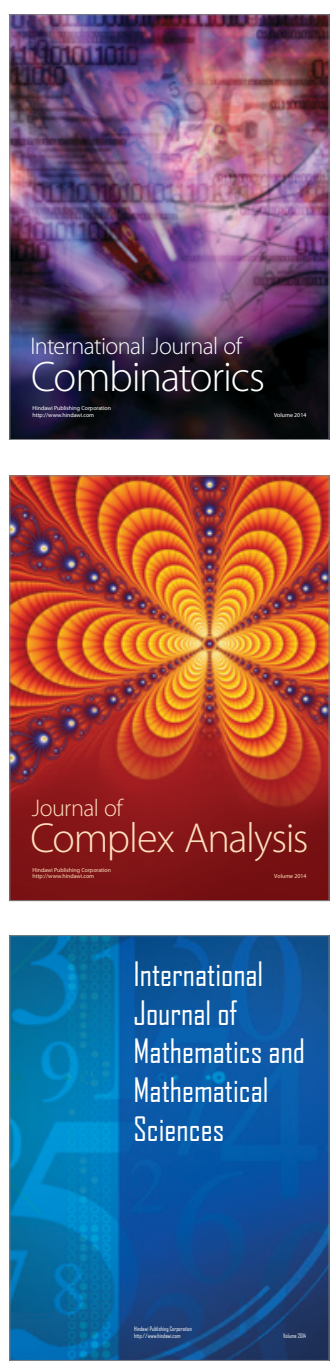
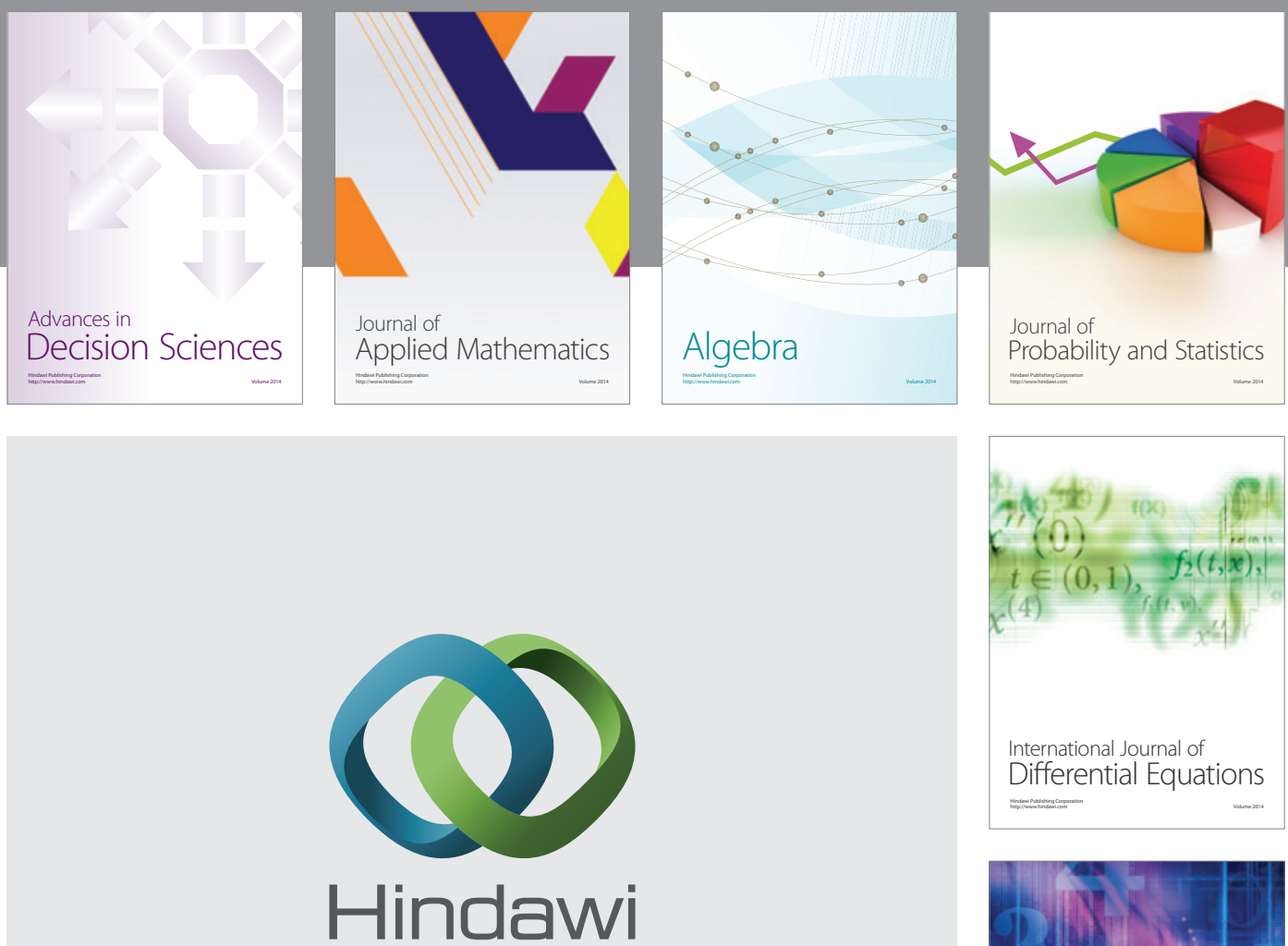

Submit your manuscripts at http://www.hindawi.com
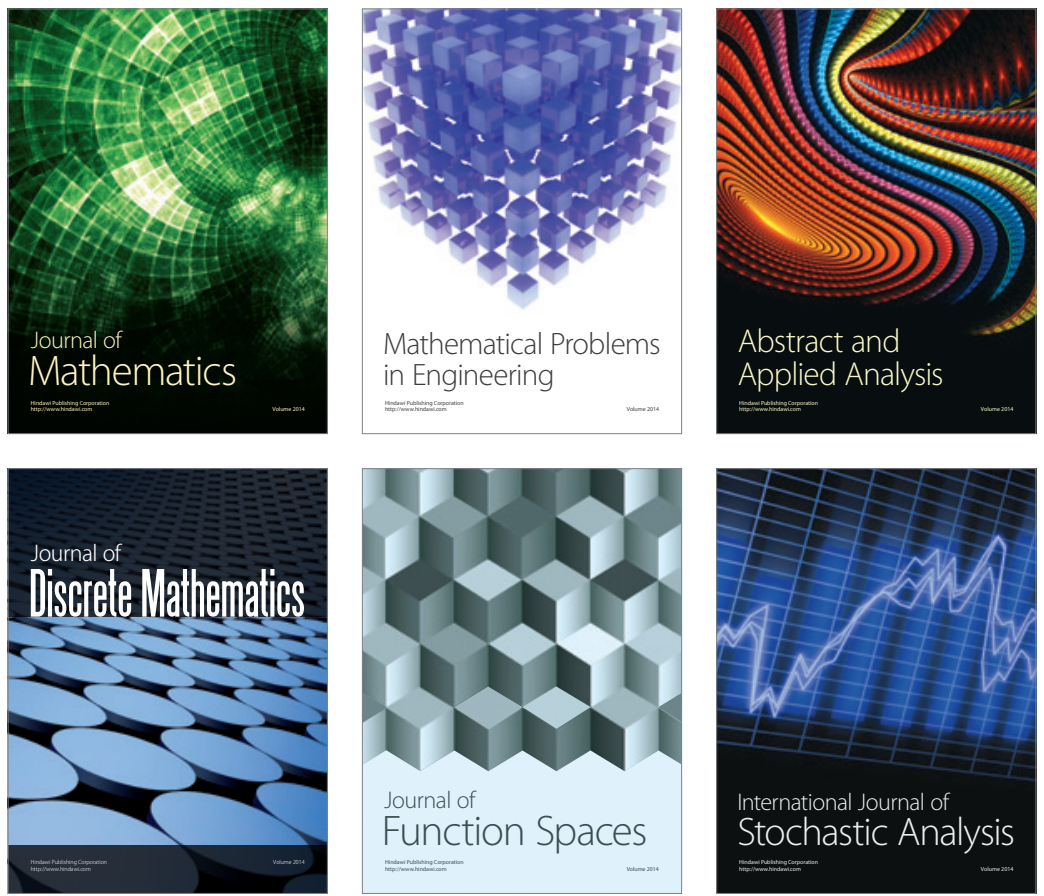

Journal of

Function Spaces

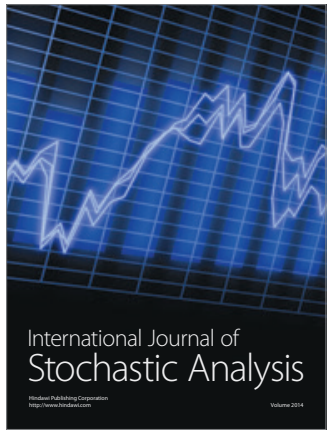

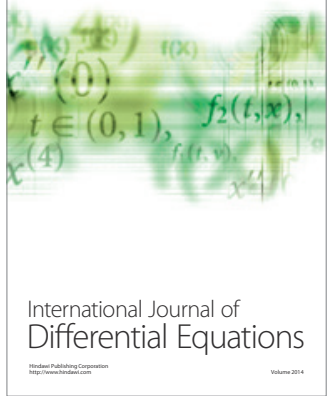
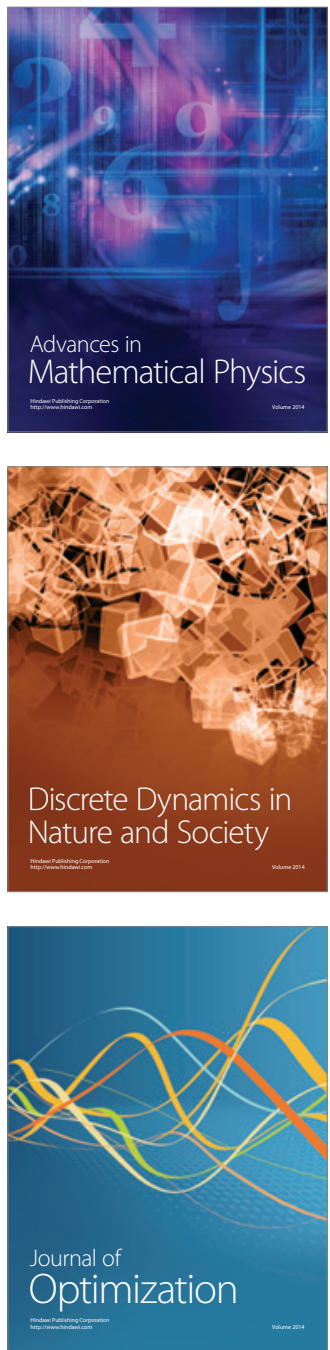